\title{
An Efficiency Maximization Design for SWIPT
}

\author{
Quang-Doanh Vu, Le-Nam Tran, Ronan Farrell, and Een-Kee Hong
}

\begin{abstract}
A joint power splitting and beamforming design for multiuser multiple-input single-output (MISO) systems where receivers have capability of decoding information and harvesting energy simultaneously from received signals is considered. The objective is to maximize the ratio of the achieved utility to the total power consumption subject to harvested power requirements and power budget at a base station (BS). The utility function of interest combines the sum rate and the total harvested power. The design problem is nonconvex, and thus, global optimality is difficult to achieve. To solve this problem locally we first convert the problem into a more tractable form, and then propose an iterative algorithm which is guaranteed to achieve a Karush-Kuhn-Tucker solution. Numerical results are provided to demonstrate the superior performance of the proposed method.
\end{abstract}

Index Terms-Energy harvesting, fractional problem, iterative algorithm, linear precoding, power splitting.

\section{INTRODUCTION}

$\mathbf{R}$ ECENTLY, simultaneous wireless information and power transfer (SWIPT) has been received growing attention in both academia and industry [1]-[9]. In this context, receivers can receive simultaneously information and energy from transmitters [5], [6]. Two common methods to design such kind of receivers are time switching and power splitting. For the former a receiver switches between being an information decoder and an energy harvester. For the latter the receiver divides the received signal into two parts, one used for decoding data and the other used for harvesting energy. In this letter we consider the power splitting mode since this method is more general than time switching [6].

Joint power splitting and precoding design for SWIPT has been the main focus of several recent works. For example, it was considered in the multiuser MISO systems in [5], [6]. These works studied the optimization problems of joint power splitting and beamforming design such that both quality of service (QoS) and harvested power constraints are satisfied with minimum

Manuscript received April 23, 2015; revised June 25, 2015; accepted July 23, 2015. Date of publication August 03, 2015; date of current version August 17, 2015. This work was supported in part by the International Research and Development Program of the National Research Foundation of Korea (NRF) funded by the Ministry of Science, ICT, and Future Planning under Grant NRF2012K1A3A1A26034927 and in part by the Irish Government and the European Union under Ireland's EU Structural and Investment Funds Programmes 2014-2020 through the SFI Research Centres Programme under Grant 13/RC/ 2077. The associate editor coordinating the review of this manuscript and approving it for publication was Dr. Jiaheng Wang.

Q.-D. Vu and E.-K. Hong are with the School of Electronics and Information, Kyung Hee University, Yongin 449-701, Korea (e-mail: vqdoanh@khu.ac.kr; ekhong@khu.ac.kr).

L.-N. Tran and R. Farrell are with the Department of Electronic Engineering, Maynooth University, Kildare, Ireland (e-mail: 1tran@eeng.nuim.ie; rfarrell@eeng.nuim.ie).

Color versions of one or more of the figures in this paper are available online at http://ieeexplore.ieee.org.

Digital Object Identifier 10.1109/LSP.2015.2464082 transmit power. While power minimization problem was well studied in the literature, energy-efficient designs for SWIPT, albeit of timely interest, have received less attention. Energyefficient designs for SWIPT were investigated in [7], [8] for two-points and three-points MIMO systems, respectively. We remark that these two schemes cannot be applied to the multiuser scenario considered herein due to the inter-user interference. The recent work in [10] considered maximizing the energy efficiency for a multiuser SWIPT system where all energy harvesters use the same fraction of power splitting.

In this letter we study the joint problem of power splitting and transmit beamforming for multiuser SWIPT with a multiple antenna base station. Different from [10], we consider a more general definition of the power usage efficiency where harvested energy is viewed as a system output. It is worth mentioning that, in the considered scenario, the system output is not only the total transmitted data but also the amount of harvested energy. Thus it would be more reasonable to introduce a utility function which blends received information with harvested energy. However, it is obvious that data rate and power have different units. Therefore, to make an appropriate combination, we introduce a utility function based on a framework of multi-objective optimization [11]. Then, to improve the efficiency of using power in the systems, the design problem is formulated such as the ratio of achieved utility to total consumed power is maximized [12]. Unfortunately, the utility function is neither concave nor convex. Consequently, the problem of interest is an intractable fractional program, and thus, it is generally difficult to achieve global optimum. In order to find a local optimal solution we first transform the original problem to an equivalent, but more tractable, form. We then introduce an iterative algorithm which successively solves approximate convex problems of the transformed problem until convergence. Moreover, we show that the obtained solutions of the proposed algorithm satisfy the necessary conditions of the considered problem. In addition, we also discuss the practical implementation of the proposed method.

Notation: Standard notations are used in this paper. Bold lower and upper case letters represent vectors and matrices, respectively; $\|\cdot\|_{2}$ represents the $l_{2}$ norm; $|\cdot|$ represents the absolute value; $\mathbb{C}^{a \times b}$ represents the space of complex matrices of dimensions given in superscript; $\mathcal{C N}(0, c)$ denotes a complex Gaussian random variable with zero mean and variance $c$; $\mathfrak{R}\{\cdot\}$ represents real part of the argument.

\section{System Model AND Problem Formulation}

Consider a multiuser MISO downlink system where a $K$-antennas BS simultaneously serves $N$ single-antenna users in the same band of frequency. Let $\mathbf{h}_{i} \in \mathbb{C}^{1 \times K}$ be the channel (row) vector from the BS to user $i$, and $\mathbf{w}_{i} \in \mathbb{C}^{K \times 1}$ be the beamformer for user $i$. Under flat fading channels, the received signal at user $i$ is written as

$$
y_{i}=\mathbf{h}_{i} \mathbf{w}_{i} x_{i}+\sum_{j=1, j \neq i}^{N} \mathbf{h}_{i} \mathbf{w}_{j} x_{j}+n_{i}
$$


where $n_{i} \sim \mathcal{C N}\left(0, \sigma_{i}^{2}\right)$ is the additive white Gaussian noise (AWGN) and $x_{i}$ is the normalized complex data symbol intended for user $i$. From (1), the total received power at user $i$ is given by [5], [6]

$$
p_{i}(\mathbf{w})=\sum_{j=1}^{N}\left|\mathbf{h}_{i} \mathbf{w}_{j}\right|^{2}+\sigma_{i}^{2}=\mathbf{w}^{H} \tilde{\mathbf{H}}_{i} \mathbf{w}+\sigma_{i}^{2}
$$

where $\mathbf{w} \triangleq\left[\mathbf{w}_{1}^{T}, \mathbf{w}_{2}^{T}, \ldots, \mathbf{w}_{N}^{T}\right]^{T} \in \mathbb{C}^{(N K \times 1)}$ and $\tilde{\mathbf{H}}_{i} \triangleq \operatorname{blkdiag}(\underbrace{\mathbf{h}_{i}^{H} \mathbf{h}_{i}, \mathbf{h}_{i}^{H} \mathbf{h}_{i}, \ldots, \mathbf{h}_{i}^{H} \mathbf{h}_{i}}_{N \text { terms }})$. In this letter, we suppose that users can harvest energy and decode information from received signals, i.e. a user splits its received signal into two parts, the first part used for data detection, the second converted to energy which is stored at the user [4], [5]. In this regard, let $0<\phi_{i}<1$ be the fraction of received power that is used for decoding information. Then, the signal-to-interference-and-noise ratio (SINR) at user $i$ is given by

$$
\begin{aligned}
\gamma_{i}\left(\phi_{i}, \mathbf{w}\right) & =\frac{\phi_{i}\left|\mathbf{h}_{i} \mathbf{w}_{i}\right|^{2}}{\phi_{i}\left(\sum_{j=1, j \neq i}^{N}\left|\mathbf{h}_{i} \mathbf{w}_{j}\right|^{2}+\sigma_{i}^{2}\right)+\eta_{i}^{2}} \\
& =\frac{\mathbf{w}_{i}^{H} \mathbf{H}_{i} \mathbf{w}_{i}}{\mathbf{w}^{H} \overline{\mathbf{H}}_{i} \mathbf{w}+\sigma_{i}^{2}+\frac{\eta_{i}^{2}}{\phi_{i}}}
\end{aligned}
$$

where $\mathbf{H}_{i} \triangleq \mathbf{h}_{i}^{H} \mathbf{h}_{i}, \overline{\mathbf{H}}_{i} \triangleq \operatorname{blkdiag}(\underbrace{\mathbf{H}_{i}, \ldots \mathbf{H}_{i}}_{i-1 \text { terms }}, \mathbf{0}, \underbrace{\mathbf{H}_{i}, \ldots \mathbf{H}_{i}}_{N-i \text { terms }})$ and $\eta_{i}^{2}$ is the variance of the additional circuit noise at user $i$, which is also modeled as zero mean AWGN [5], [6]. The harvested power at user $i$ is given by

$$
p_{i}^{\mathrm{eh}}\left(\phi_{i}, \mathbf{w}\right)=\alpha_{i}\left(1-\phi_{i}\right) p_{i}(\mathbf{w})
$$

where $\alpha_{i} \in(0,1)$ is the parameter of energy harvesting efficiency of user $i$. In this work, we jointly find beamforming vectors $\mathbf{w}$ and fractions of received power $\left\{\phi_{i}\right\}$ such that power is used efficiently. To measure the efficiency of the power consumed in a SWIPT system, we introduce a utility function as well as a performance measure as follows.

Utility function and performance measure: As mentioned earlier, the utility function should capture both system throughput and harvested power to produce a more precise measurement on the power consumption efficiency. To deal with the different dimensions of the two quantities, we introduce a utility function based on the multi-objective optimization approach [11]. For this purpose let $r_{0}>0$ and $p_{0}>0$ be the reference data rate and power, respectively. Then the utility function is written as

$$
\begin{aligned}
f_{\mathrm{u}}(\boldsymbol{\phi}, \mathbf{w})= & b \frac{\sum_{i=1}^{N} \log \left(1+\gamma_{i}\left(\phi_{i}, \mathbf{w}\right)\right)}{r_{0}} \\
& +(1-b) \frac{\sum_{i=1}^{N} p_{i}^{\mathrm{eh}}\left(\phi_{i}, \mathbf{w}\right)}{p_{0}}
\end{aligned}
$$

where $b \in[0,1]$ is the priority parameter and $\phi \triangleq\left\{\phi_{i}\right\}$. It is proper to quantify the efficiency of using power by the ratio of utility to total consumed power [12]. In this work we model the amount of consumed power as

$$
f_{\mathrm{c}}(\mathbf{w})=p^{\mathrm{cir}}+\frac{1}{\beta}\|\mathbf{w}\|_{2}^{2}
$$

where $p^{\text {cir }}$ is the circuit power, which is assumed to be independent from data rate, and $\beta \in(0,1)$ is the amplifier efficiency of the BS.
Based on the above introduced notations and discussions, the problem of joint power splitting and beamformer design for maximizing the efficiency of using power in the considered system is formulated as

$$
\begin{array}{cl}
\underset{\phi, \mathbf{w}}{\operatorname{maximize}} & f(\boldsymbol{\phi}, \mathbf{w}) \triangleq \frac{f_{\mathrm{u}}(\boldsymbol{\phi}, \mathbf{w})}{f_{\mathrm{c}}(\mathbf{w})} \\
\text { subject to } & p_{i}^{\mathrm{eh}}\left(\phi_{i}, \mathbf{w}\right) \geq \bar{p}_{i}, \forall i \\
& 0<\phi_{i}<1, \forall i \\
& \|\mathbf{w}\|_{2}^{2} \leq \bar{P}
\end{array}
$$

where $\bar{p}_{i}$ 's are predefined thresholds of harvested power and $\bar{P}$ is the maximum transmit power at the BS. We note that finding an optimal solution to (7) is challenging due to the nonconvexity of the objective and the feasible set. In the following section we propose an iterative method that arrives at a stationary point of (7).

\section{Proposed Method}

The proposed iterative algorithm is based on the inner approximation method in [13]. The idea is to transform (7) into an equivalent formulation in which its convexity is more exposed, and then apply convex approximation to the nonconvex parts. This technique has been widely used in wireless communication designs, e.g. recently in [14]. We first introduce new optimization variables $\boldsymbol{\nu}=\left\{\nu_{i}\right\}, \mathbf{z}=\left\{z_{i}\right\}$, and $\mathbf{s}=\left\{s_{i}\right\}$, and rewrite (7) equivalently as

$$
\begin{array}{cl}
\underset{\phi, \mathbf{w}, \boldsymbol{\nu}, \mathbf{z}, \mathbf{s}}{\operatorname{maximize}} & \frac{\sum_{i=1}^{N} \tilde{r}_{0} z_{i}+\tilde{p}_{0} s_{i}}{p^{\text {cir }}+\frac{1}{\beta}\|\mathbf{w}\|_{2}^{2}} \\
\text { subject to } & \gamma_{i}\left(\phi_{i}, \mathbf{w}\right) \geq \nu_{i}, \forall i \\
& p_{i}^{\text {eh }}\left(\phi_{i}, \mathbf{w}\right) \geq s_{i}, \forall i \\
& \log \left(1+\nu_{i}\right) \geq z_{i}, \forall i \\
& s_{i} \geq \bar{p}_{i}, \forall i \\
& (7 \mathrm{c}),(7 \mathrm{~d})
\end{array}
$$

where $\tilde{r}_{0} \triangleq \frac{b}{r_{0}}$ and $\tilde{p}_{0} \triangleq \frac{(1-b)}{p_{0}}$. The equivalence between (7) and (8) is due to the fact that all the constraints from (8b) to (8d) hold with equality at optimality. To see this suppose that (8b) is not active at optimality, i.e., $\gamma_{i}\left(\phi_{i}, \mathbf{w}\right)>\nu_{i}$ for some $i$. Then there exist $\Delta \nu_{i}>0$ and $\Delta z_{i}>0$ such that $\gamma_{i}\left(\phi_{i}, \mathbf{w}\right)=$ $\nu_{i}+\Delta \nu_{i}$ and $\log \left(1+\nu_{i}+\Delta \nu_{i}\right)=z_{i}+\Delta z_{i}$. Thus $z_{i}+\Delta z_{i}$ is also feasible to (8) but results in a strictly larger objective. This contradicts with the optimality assumption. Using (3) and (4) we can further rewrite problem (8) explicitly as

$$
\begin{array}{ll}
\underset{\phi, \mathbf{w}, \boldsymbol{\nu}, \mathbf{z}, \mathbf{s}}{\operatorname{maximize}} & \frac{\sum_{i=1}^{N} \tilde{r}_{0} z_{i}+\tilde{p}_{0} s_{i}}{p^{\mathrm{cir}}+\frac{1}{\beta}\|\mathbf{w}\|_{2}^{2}} \\
\text { subject to } & \frac{\mathbf{w}_{i}^{H} \mathbf{H}_{i} \mathbf{w}_{i}}{\nu_{i}} \geq \mathbf{w}^{H} \overline{\mathbf{H}}_{i} \mathbf{w}+\sigma_{i}^{2}+\frac{\eta_{i}^{2}}{\phi_{i}}, \forall i \\
& \frac{\mathbf{w}^{H} \tilde{\mathbf{H}}_{i} \mathbf{w}+\sigma_{i}^{2}}{s_{i}} \geq \frac{1}{\alpha_{i}\left(1-\phi_{i}\right)}, \forall i \\
& (8 \mathrm{~d}),(8 \mathrm{e}),(8 \mathrm{f}) .
\end{array}
$$

Although the formulation in (9) is still intractable, it is now easier to deal with the nonconvex parts of the problem. In this regard, two remarks are in order. First, the objective in (9a) is a ratio between an affine function and a quadratic one. Second, the constraints in (9d) are convex, while those in (9b) and (9c) are nonconvex. A more important observation is that the functions in both sides of the constraints in (9b) and (9c) are convex, 
which is useful for developing an approximate solution for (9). We recall that quadratic-over-linear function, e.g. $\frac{\mathbf{w}_{i}^{H} \mathbf{H}_{i} \mathbf{w}_{i}}{\nu_{i}}$, is convex [15, Ch. 3].

We now present a way to reduce the objective (9a) into a linear function without affecting the type of the constraints in (9). Specifically, by applying Charnes-Cooper's transformation [16], we have the following theorem whose proof can be found in the Appendix.

Theorem 1: Let $\left(\boldsymbol{\phi}^{*}, \mathbf{w}^{*}, \boldsymbol{\nu}^{*}, \mathbf{z}^{*}, \mathbf{s}^{*}\right)$ be an optimal of (9), then $\left(\tilde{\boldsymbol{\phi}}=\boldsymbol{\phi}^{*} / f_{\mathrm{c}}\left(\mathbf{w}^{*}\right), \tilde{\mathbf{w}}=\mathbf{w}^{*} / f_{\mathrm{c}}\left(\mathbf{w}^{*}\right), \tilde{\boldsymbol{\nu}}=\boldsymbol{\nu}^{*} / f_{\mathrm{c}}\left(\mathbf{w}^{*}\right)\right.$, $\left.\tilde{\mathbf{z}}=\mathbf{z}^{*} / f_{\mathrm{c}}\left(\mathbf{w}^{*}\right), \tilde{\mathbf{s}}=\mathbf{s}^{*} / f_{\mathrm{c}}\left(\mathbf{w}^{*}\right), t=1 / f_{\mathrm{c}}\left(\mathbf{w}^{*}\right)\right)$ is an optimal of the following problem

$$
\begin{array}{ll}
\underset{\tilde{\boldsymbol{\phi}}, \tilde{\mathbf{w}}, \tilde{\boldsymbol{\nu}}, \tilde{\mathbf{z}}, \tilde{\mathbf{s}}, t}{\operatorname{maximize}} & \sum_{i=1}^{N} \tilde{r}_{0} \tilde{z}_{i}+\tilde{p}_{0} \tilde{s}_{i} \\
\text { subject to } & t^{2} p^{\text {cir }}+\frac{1}{\beta}\|\tilde{\mathbf{w}}\|_{2}^{2} \leq t \\
& \frac{\tilde{\mathbf{w}}_{i}^{H} \mathbf{H}_{i} \tilde{\mathbf{w}}_{i}}{\tilde{\nu}_{i}} \geq \frac{\tilde{\mathbf{w}}^{H} \overline{\mathbf{H}}_{i} \tilde{\mathbf{w}}}{t} \\
& +t \sigma_{i}^{2}+\eta_{i}^{2} \frac{t^{2}}{\tilde{\phi}_{i}}, \forall i \\
& \frac{\tilde{\mathbf{w}}^{H} \tilde{\mathbf{H}}_{i} \tilde{\mathbf{w}}+t^{2} \sigma_{i}^{2}}{\tilde{s}_{i}} \geq \frac{t^{2}}{\alpha_{i}\left(t-\tilde{\phi}_{i}\right)}, \forall i \\
& t \log \left(1+\tilde{\nu}_{i} / t\right) \geq \tilde{z}_{i}, 0<\tilde{\phi}_{i}<t, \forall i \\
& \tilde{s}_{i} \geq t \bar{p}_{i}, \forall i \\
& \|\tilde{\mathbf{w}}\|_{2} \leq t \sqrt{\bar{P}} .
\end{array}
$$

On the other hand, let $\left(\tilde{\boldsymbol{\phi}}^{*}, \tilde{\mathbf{w}}^{*}, \tilde{\boldsymbol{\nu}}^{*}, \tilde{\mathbf{z}}^{*}, \tilde{\mathbf{s}}^{*}, t^{*}\right)$ be an optimal of (10), then $\left(\boldsymbol{\phi}=\tilde{\boldsymbol{\phi}}^{*} / t^{*}, \mathbf{w}=\tilde{\mathbf{w}}^{*} / t^{*}, \boldsymbol{\nu}=\tilde{\boldsymbol{\nu}}^{*} / t^{*}, \mathbf{z}=\tilde{\mathbf{z}}^{*} / t^{*}\right.$, $\left.\mathbf{s}=\tilde{\mathbf{s}}^{*} / t^{*}\right)$ is an optimal of (9).

By Theorem 1 we now focus on solving (10), which has a simpler form in the sense that the nonconvexity of the objective in (9) is eliminated. To deal with these constraints in (10c) and (10d) we develop an iterative procedure which solves (10) locally. In particular, at iteration $n+1$, we solve the following convex problem

$$
\begin{array}{cc}
\underset{\tilde{\boldsymbol{\phi}}, \tilde{\mathbf{w}}, \tilde{\boldsymbol{\nu}}, \tilde{\mathbf{z}}, \tilde{\mathbf{s}}, t}{\operatorname{maximize}} \quad \sum_{i=1}^{N} \tilde{r}_{0} \tilde{z}_{i}+\tilde{p}_{0} \tilde{s}_{i} \\
\text { subject to } \quad F_{i}^{(n)}\left(\tilde{\mathbf{w}}_{i}, \tilde{\nu}_{i}\right) \geq \frac{\tilde{\mathbf{w}}^{H} \overline{\mathbf{H}}_{i} \tilde{\mathbf{w}}}{t} \\
+t \sigma_{i}^{2}+\eta_{i}^{2} \frac{t^{2}}{\tilde{\phi}_{i}}, \forall i \\
G_{i}^{(n)}\left(\tilde{\mathbf{w}}, \tilde{s}_{i}, t\right) \geq \frac{t^{2}}{\alpha_{i}\left(t-\tilde{\phi}_{i}\right)}, \forall i \\
(10 \mathrm{~b}),(10 \mathrm{e}),(10 \mathrm{f}),(10 \mathrm{~g})
\end{array}
$$

where $F_{i}^{(n)}\left(\tilde{\mathbf{w}}_{i}, \tilde{\nu}_{i}\right) \triangleq 2 \Re\left\{\left(\tilde{\mathbf{w}}_{i}^{(n)}\right)^{H} \mathbf{H}_{i} \tilde{\mathbf{w}}_{i}\right\}\left(\tilde{\nu}_{i}^{(n)}\right)^{-1}-$ $\left(\tilde{\mathbf{w}}_{i}^{(n)}\right)^{H} \mathbf{H}_{i} \tilde{\mathbf{w}}_{i}^{(n)}\left(\tilde{\nu}_{i}^{(n)}\right)^{-2} \tilde{\nu}_{i}$ and $G_{i}^{(n)}\left(\tilde{\mathbf{w}}, \tilde{s}_{i}, t\right) \triangleq$ $2\left(\Re\left\{\left(\tilde{\mathbf{w}}^{(n)}\right)^{H} \tilde{\mathbf{H}}_{i} \tilde{\mathbf{w}}\right\}+\sigma_{i}^{2} t^{(n)} t\right)\left(\tilde{s}_{i}^{(n)}\right)^{-1}-\left(\left(\tilde{\mathbf{w}}^{(n)}\right)^{H} \tilde{\mathbf{H}}_{i} \tilde{\mathbf{w}}^{(n)}+\right.$ $\left.\sigma_{i}^{2}\left(t^{(n)}\right)^{2}\right)\left(\tilde{s}_{i}^{(n)}\right)^{-2} \tilde{s}_{i}$ are the first-order approximation of $\frac{\tilde{\mathbf{w}}_{i}^{H} \mathbf{H}_{i} \tilde{\mathbf{w}}_{i}}{\tilde{\boldsymbol{\nu}}_{i}}$ and $\frac{\tilde{\mathbf{w}}^{H} \tilde{\mathbf{H}}_{i} \tilde{\mathbf{w}}+t^{2} \sigma_{i}^{2}}{\tilde{\boldsymbol{s}}_{i}}$ around the point $\left(\tilde{\boldsymbol{\phi}}^{(n)}, \tilde{\mathbf{w}}^{(n)}, \tilde{\boldsymbol{\nu}}^{(n)}, \tilde{\mathbf{z}}^{(n)}, \tilde{\mathbf{s}}^{(n)}, t^{(n)}\right)^{\tilde{s}_{i}}$, respectively.

We outline the proposed method in Algorithm 1. By a result in [13], the iterative procedure of Algorithm 1 (i.e., steps 2-6) provably converges to a Karush-Kuhn-Tucker (KKT) solution of (10). Due to Theorem 1, it is not difficult to see that the output of Algorithm 1 is a stationary point of (7). We omit the details for the sake of brevity. We now provide the complexity analysis of the proposed algorithm. Specifically, in each iteration of Algorithm 1, the worst-case computational complexity for solving the generic convex problem in (11) using interior point methods is given by $\mathcal{O}\left((K N+4 N+1)^{4}\right)=\mathcal{O}\left((K+4)^{4} N^{4}\right)[17$, Chap. $6]$.

\section{Algorithm 1 The proposed algorithm to solve (7)}

1: Initialization: set $n:=0$ and generate initial feasible point $\left(\tilde{\boldsymbol{\phi}}^{(0)}, \tilde{\mathbf{w}}^{(0)}, \tilde{\boldsymbol{\nu}}^{(0)}, \tilde{\mathbf{z}}^{(0)}, \tilde{\mathbf{s}}^{(0)}, t^{(0)}\right)$.

\section{2: repeat}

3: $\quad$ Solve (11) to obtain optimal values

$\left(\tilde{\boldsymbol{\phi}}^{*}, \tilde{\mathbf{w}}^{*}, \tilde{\boldsymbol{\nu}}^{*}, \tilde{\mathbf{z}}^{*}, \tilde{\mathbf{s}}^{*}, t^{*}\right)$.

4: $\quad \operatorname{Set}\left(\tilde{\boldsymbol{\phi}}^{(n+1)}, \tilde{\mathbf{w}}^{(n+1)}, \tilde{\boldsymbol{\nu}}^{(n+1)}, \tilde{\mathbf{z}}^{(n+1)}, \tilde{\mathbf{s}}^{(n+1)}, t^{(n+1)}\right)$ $:=\left(\tilde{\phi}^{*}, \tilde{\mathbf{w}}^{*}, \tilde{\boldsymbol{\nu}}^{*}, \tilde{\mathbf{z}}^{*}, \tilde{\mathbf{s}}^{*}, t^{*}\right)$.

5: $\quad$ Update $n:=n+1$.

\section{6: until Convergence}

7: $\quad$ Output: $\mathbf{w}=\tilde{\mathbf{w}}^{(n)} / t^{(n)}$ and $\boldsymbol{\phi}=\tilde{\boldsymbol{\phi}}^{(n)} / t^{(n)}$

Practical Considerations: Algorithm 1 (step 1) requires a feasible point of (10) to start, which is difficult to find in general. To overcome this problem we now present a variant of Algorithm 1, which is based on the idea introduced in [18]. We begin modifying (10) into the following formulation

$$
\begin{aligned}
\underset{\tilde{\boldsymbol{\phi}}, \tilde{\mathbf{w}}, \tilde{\boldsymbol{\nu}}, \tilde{\mathbf{z}}, \tilde{\mathbf{s}}, t, \mathbf{u}}{\operatorname{maximize}} & \sum_{i=1}^{N} \tilde{r}_{0} \tilde{z}_{i}+\tilde{p}_{0} \tilde{s}_{i}+u_{i} \\
\text { subject to } & \tilde{s}_{i} \geq t \bar{p}_{i}+u_{i}, \forall i \\
& u_{i} \leq 0, \forall i \\
& (10 \mathrm{~b}),(10 \mathrm{c}),(10 \mathrm{~d}),(10 \mathrm{e}),(10 \mathrm{~g})
\end{aligned}
$$

where $\mathbf{u}=\left[u_{1}, u_{2}, \ldots, u_{N}\right]$ are newly introduced variables and can be viewed as regulated terms added to (10f). Note that if $\mathbf{u}=\mathbf{0}$ then $(\tilde{\boldsymbol{\phi}}, \tilde{\mathbf{w}}, \tilde{\boldsymbol{\nu}}, \tilde{\mathbf{z}}, \tilde{\mathbf{s}}, t)$ is also feasible to (10).

A feasible point of (12) can be found easily as follows. We first randomly generate $\boldsymbol{\phi}$ over the interval $(\mathbf{0}, \mathbf{1})$ and $\mathbf{w}$ over $\mathbb{C}^{(N K \times 1)}$, and then scale $\mathbf{w}$ properly to satisfy $\|\mathbf{w}\|_{2}^{2} \leq \bar{P}$. Then $\boldsymbol{\nu}, \mathbf{s}$, and $\mathbf{z}$ are computed by setting the constraints in (8b)-(8d) to be equality, respectively. Using the transformation in Theorem 1 we arrive at $(\tilde{\boldsymbol{\phi}}, \tilde{\mathbf{w}}, \tilde{\boldsymbol{\nu}}, \tilde{\mathbf{z}}, \tilde{\mathbf{s}}, t)$. In this way the constraints in (12d) are satisfied automatically. Moreover, the constraints in (12b) and (12c) can be met by choosing sufficiently small $\mathbf{u}$. From this feasible point $(\tilde{\boldsymbol{\phi}}, \tilde{\mathbf{w}}, \tilde{\boldsymbol{\nu}}, \tilde{\mathbf{z}}, \tilde{\mathbf{s}}, t, \mathbf{u})$ we apply the iterative procedure similar to Algorithm 1 to solve (12). In some first iterations, $\mathbf{u}$ can be strictly smaller than zero, but they are encouraged to be zero due to the maximization in (12a). We numerically observe it usually takes no more than 2 iterations to output a feasible point of (10). When $\mathbf{u}=\mathbf{0}$, we omit $\mathbf{u}$ and start Algorithm 1 from the obtained $(\tilde{\boldsymbol{\phi}}, \tilde{\mathbf{w}}, \tilde{\boldsymbol{\nu}}, \tilde{\mathbf{z}}, \tilde{\mathbf{s}}, t)$ which is now feasible to (10).

\section{NumericAl Results}

In this section we numerically evaluate the performance of Algorithm 1. We consider a simulation model based on the settings in [4]-[6], [19]. Particularly, the path loss model is $\sigma_{\mathrm{PL}}(\mathrm{dB})=30.18+26 \log _{10}(d)$ where $d$ is the distance in meters [20]. Near-BS users are considered, i.e. $d \in(20,30) \mathrm{m}$. 


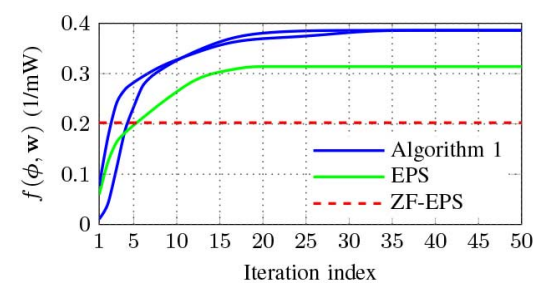

Fig. 1. Convergence behavior of Algorithm 1 with different initial points over a random channel. $N=6, K=10$, and $b=0.5$.

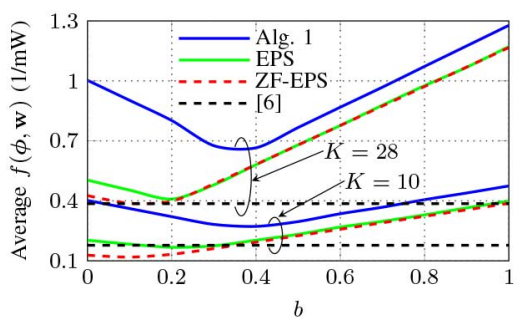

Fig. 2. Average $f(\phi, \mathbf{w})$ performances of the considered schemes versus the priority parameter $b . N=4$, and $K=\{10,28\}$.

The small-scale fading is modeled as $\mathcal{C N}(0,1)$ and the bandwidth is $1 \mathrm{MHz}$. The noise variances are $\sigma_{i}^{2}=-80 \mathrm{dBm}$, $\eta_{i}^{2}=-70 \mathrm{dBm}$ and the harvested power requirements are $\bar{p}_{i}=\bar{p}=-55 \mathrm{dBm}$, for all $i$. Reference data rate and power are simply set as $r_{0}=1(\mathrm{Mbits} / \mathrm{s})$ and $p_{0}=\bar{p}$, respectively. The power budget at BS is $\bar{P}=32 \mathrm{dBm}$, the total circuit power is $p^{\text {cir }}=5 \mathrm{dBm}$, and the amplifier efficiency of the $\mathrm{BS}$ is $\beta=0.69$. The energy harvesting efficiency of users are $\alpha_{i}=\alpha=0.45$, for all $i$. For benchmarking purpose we compare Algorithm 1 with two other suboptimal methods, which are referred to as equal power splitting (EPS) and zero-forcing EPS (ZF-EPS). In the former method the power splitting coefficients are fixed to be $\phi_{i}=0.5, \forall i$ and the beamformer vector $\mathbf{w}$ is found using an iterative method similar to Algorithm 1. In the latter scheme, in addition to fixing $\phi_{i}=0.5, \forall i$, zero-forcing beamforming technique is assumed to eliminate inter-user interference. Accordingly, the resulting problem is a concave-convex fractional program which can be solved exactly by e.g. Dinkelbach's method [21]. To solve convex problems we use the modeling package CVX [22] with SDPT3 [23] being the internal solver.

In the first experiment we investigate the convergence behavior of the proposed algorithm over a random channel with different initial points. Performances of ZF-EPS and EPS are also plotted. As can be seen in Fig. 1, Algorithm 1 converges within tens of iterations in all cases. We remark that for the first two iterations not included in Fig. 1, the obtained solutions are infeasible to (10) since Algorithm 1 starts with the regulated formulation in (12). Despite different initial points Algorithm 1 converges to the same solution. We also see that EPS converges faster, but to a point inferior to the one obtained by Algorithm 1 . The reason for this fact is that optimal $\phi$ is not considered in EPS.

Fig. 2 depicts the average $f(\phi, \mathbf{w})$ performances versus the priority parameter $b .{ }^{1}$ In this figure, the solution presented in [6], which considered the power minimization problem with both rate and harvested power constraints, is also plotted. To

\footnotetext{
${ }^{1}$ We note that when $b=1$ the proposed problem becomes the traditional energy-efficiency maximization [12].
}

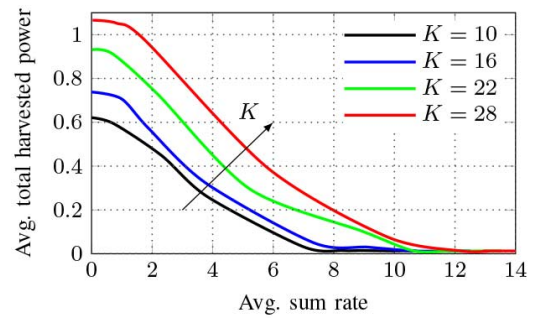

Fig. 3. The trade-off between sum rate (Mnats/s) and total harvested power $(\mu \mathrm{W})$ with different number of transmit antenna. $N=4$.

achieve a fair comparison we include user-specific data rate requirements in other schemes by simply adding linear constraints $z_{i} \geq r_{0}, \forall i$. As can be seen, the proposed design shows superior performance compared to the others in all cases. Another interesting observation is that, with large value of $b$, the performance of ZF-EPS is lower than that of EPS when $K=10$, and these two schemes achieve almost the same performance when $K=28$. The reason is that, when $b$ is large, data rate takes much more effect on the utility function than harvested power, and it is also known that zero-forcing technique yields good data rate performance for a large number of transmit antennas.

In Fig. 3 we plot the trade-off between achieved sum rate and total harvested power. This is accomplished by varying $b$ over interval [0,1]. An expected observation shown in Fig. 3 is that, with respect to $b$, the sum rate is increasing and the total harvested power is decreasing. In particular, the total harvested power converges to $\sum_{i=1}^{N} \bar{p}_{i}$ when $b=1$, and sum rate is zero when $b=0$ due to rate requirements are not considered. Another interesting result is that both sum rate and total harvested power increase when number of transmit antenna increases. This improvement implies that the proposed algorithm is able to exploit available degree of freedom offered by the increased number of transmit antennas.

\section{CONCLUSION}

In this letter a MISO system consisting of multiple users who have capability of decoding information and harvesting energy simultaneously has been studied. We develop an iterative algorithm which jointly designs power splitting and beamforming to maximize power efficiency in the system. Moreover, the proposed algorithm is guaranteed to achieve a stationary point of the nonconvex design problem. Simulation results demonstrate the superior performance of the proposed algorithm compared to the known solutions.

\section{APPENDIX}

Let $\mathcal{S}$ and $\tilde{\mathcal{S}}$ be feasible sets of (9) and (10), respectively. Consider a point $(\boldsymbol{\phi}, \mathbf{w}, \boldsymbol{\nu}, \mathbf{z}, \mathbf{s}) \in \mathcal{S}$. From (6), we can see that $f_{\mathrm{c}}(\mathbf{w})>0$ for all $\mathbf{w} \in \mathbb{C}^{K N \times 1}$. Thus the mapping from $\mathcal{S}$ to $\tilde{\mathcal{S}}$, i.e., $\tilde{\boldsymbol{\phi}}=\phi / f_{\mathrm{c}}(\mathbf{w}), \tilde{\mathbf{w}}=\mathbf{w} / f_{\mathrm{c}}(\mathbf{w})$, $\tilde{\boldsymbol{\nu}}=\boldsymbol{\nu} / f_{\mathrm{c}}(\mathbf{w}), \tilde{\mathbf{z}}=\mathbf{z} / f_{\mathrm{c}}(\mathbf{w}), \tilde{\mathbf{s}}=\mathbf{s} / f_{\mathrm{c}}(\mathbf{w})$, and $t=1 / f_{\mathrm{c}}(\mathbf{w})$, is well defined. Further it is not difficult to verify that $(\tilde{\boldsymbol{\phi}}, \tilde{\mathbf{w}}, \tilde{\boldsymbol{\nu}}, \tilde{\mathbf{z}}, \tilde{\mathbf{s}}, t) \in \tilde{\mathcal{S}}$ attains the same objective, i.e. $\sum_{i=1}^{N} \tilde{r}_{0} \tilde{z}_{i}+\tilde{p}_{0} \tilde{s}_{i}=\frac{\sum_{i=1}^{N} \tilde{r}_{0} z_{i}+\tilde{p}_{0} s_{i}}{p^{\mathrm{cir}}+\frac{1}{\beta}\|\mathbf{w}\|_{2}^{2}}$. Similarly if $(\tilde{\boldsymbol{\phi}}, \tilde{\mathbf{w}}, \tilde{\boldsymbol{\nu}}, \tilde{\mathbf{z}}, \tilde{\mathbf{s}}, t) \in \tilde{\mathcal{S}}$ then it is obvious that the mapping $(\tilde{\boldsymbol{\phi}}, \tilde{\mathbf{w}}, \tilde{\boldsymbol{\nu}}, \tilde{\mathbf{z}}, \tilde{\mathbf{s}}, t) \in \tilde{\mathcal{S}} \rightarrow(\tilde{\boldsymbol{\phi}} / t, \tilde{\mathbf{w}} / t, \tilde{\boldsymbol{\nu}} / t, \tilde{\mathbf{z}} / t, \tilde{\mathbf{s}} / t) \in \mathcal{S} \quad$ also yields the same objective. In other words, the Charnes-Cooper's transformation is a one-to-one mapping between $\mathcal{S}$ and $\tilde{\mathcal{S}}$ with the same objective, which completes the proof. 


\section{REFERENCES}

[1] P. Grover and A. Sahai, "Shannon meets tesla: Wireless information and power transfer," in Proc. IEEE Int. Symp. Inf. Theory (ISIT), Jun. 2010, pp. 2363-2367.

[2] O. Ozel, K. Tutuncuoglu, J. Yang, S. Ulukus, and A. Yener, "Transmission with energy harvesting nodes in fading wireless channels: Optimal policies," IEEE J. Sel. Areas Commun., vol. 29, no. 8, pp. 1732-1743, Sep. 2011.

[3] D. Ng, E. Lo, and R. Schober, "Energy-efficient resource allocation in multiuser OFDM systems with wireless information and power transfer," in Proc. IEEE Wireless Commun. Netw. Conf. (WCNC), Apr. 2013, pp. 3823-3828.

[4] R. Zhang and C. K. Ho, "MIMO broadcasting for simultaneous wireless information and power transfer," IEEE Trans. Wireless Commun., vol. 12, no. 5, pp. 1989-2001, May 2013.

[5] S. Timotheou, I. Krikidis, G. Zheng, and B. Ottersten, "Beamforming for MISO interference channels with QoS and RF energy transfer," IEEE Trans. Wireless Commun., vol. 13, no. 5, pp. 2646-2658, May 2014.

[6] Q. Shi, L. Liu, W. Xu, and R. Zhang, "Joint transmit beamforming and receive power splitting for MISO SWIPT systems," IEEE Trans. Wireless Commun., vol. 13, no. 6, pp. 3269-3280, Jun. 2014.

[7] X. Chen, X. Wang, and X. Chen, "Energy-efficient optimization for wireless information and power transfer in large-scale MIMO systems employing energy beamforming," IEEE Wireless Commun. Lett., vol. 2, no. 6, pp. 667-670, Dec. 2013.

[8] Q. Sun, L. Li, and J. Mao, "Simultaneous information and power transfer scheme for energy efficient mimo systems," IEEE Commun. Lett., vol. 18, no. 4, pp. 600-603, Apr. 2014.

[9] K. Huang and V. Lau, "Enabling wireless power transfer in cellular networks: Architecture, modeling and deployment," IEEE Trans. Wireless Commun., vol. 13, no. 2, pp. 902-912, Feb. 2014.

[10] S. He, Y. Huang, W. Chen, S. Jin, H. Wang, and L. Yang, "Energy efficient coordinated precoding design for a multicell system with RF energy harvesting," EURASIP J. Wireless Commun. Netw., Mar. 2015, DOI 10.1186/s13638-015-0299-0.

[11] R. Marler and J. Arora, "Survey of multi-objective optimization methods for engineering," Structural Multidisciplinary Optimiz., vol. 26, pp. 369-395, Apr. 2004.
[12] C. Isheden, Z. Chong, E. Jorswieck, and G. Fettweis, "Framework for link-level energy efficiency optimization with informed transmitter," IEEE Trans. Wireless Commun., vol. 11, no. 8, pp. 2946-2957, Aug. 2012.

[13] B. R. Marks and G. P. Wright, "A general inner approximation algorithm for nonconvex mathematical programs," Operat. Res., vol. 26, no. 4, pp. 681-683, Aug. 1978.

[14] D. Nguyen, L.-N. Tran, P. Pirinen, and M. Latva-aho, "On the spectral efficiency of full-duplex small cell wireless systems," IEEE Trans. Wireless Commun., vol. 13, no. 9, pp. 4896-4910, Sep. 2014.

[15] S. Boyd and L. Vandenberghe, Convex Optimization, S. Boyd, Ed., 1st ed. Cambridge, U.K.: Cambridge Univ. Press, 2004.

[16] A. Charnes and W. W. Cooper, "Programming with linear fractional functionals," Naval Res. Logist. Quart., vol. 9, no. 3-4, pp. 181-186, 1962.

[17] A. Ben-Tal and A. Nemirovski, "Lectures on modern convex optimization," Soc. Ind. Appl. Math. (SIAM), 2001.

[18] O. Mehanna, K. Huang, B. Gopalakrishnan, A. Konar, and N. Sidiropoulos, "Feasible point pursuit and successive approximation of non-convex QCQPs," IEEE Signal Process. Lett., vol. 22, no. 7, pp. 804-808, Jul. 2015.

[19] Y. Cui, V. Lau, and Y. Wu, "Delay-aware BS discontinuous transmission control and user scheduling for energy harvesting downlink coordinated MIMO systems," IEEE Trans. Signal Process., vol. 60, no. 7, pp. 3786-3795, Jul. 2012.

[20] 3GPP, "Spatial channel model for multiple input multiple output (MIMO) simulations," 3rd Generation Partnership Project, TR 25.996 [Online]. Available: http://www.3gpp.org/technologies

[21] W. Dinkelbach, "On nonlinear fractional programming," Manage. Sci., vol. 13 , no. 7 , pp. 492-498, Oct. 1967.

[22] M. Grant and S. Boyd, CVX: Matlab software for disciplined convex programming, version 2.0 beta. Sep. 2012 [Online]. Available: http:// cvxr.com/cvx

[23] K.-C. Toh, M. J. Todd, and R. H. Tütüncü, On the implementation and usage of SDPT3-a MATLAB software package for semidefinitequadratic-linear programming,version 4.0. 2010 [Online]. Available: http://hdl.handle.net/1813/15133 